PROCEEDINGS OF THE

AMERICAN MATHEMATICAL SOCIETY

Volume 133, Number 11, Pages 3333-3339

S 0002-9939(05)08154-2

Article electronically published on May 4, 2005

\title{
CESÀRO SUMMABILITY IN A LINEAR AUTONOMOUS DIFFERENCE EQUATION
}

\author{
MIHÁLY PITUK \\ (Communicated by Carmen C. Chicone)
}

\begin{abstract}
For a linear autonomous difference equation with a unique real eigenvalue $\lambda_{0}$, it is shown that for every solution $x$ the ratio of $x$ and the eigensolution corresponding to $\lambda_{0}$ is Cesàro summable to a limit which can be expressed in terms of the initial data. As a consequence, for most solutions the Lyapunov characteristic exponent is equal to $\lambda_{0}$. The proof is based on a Tauberian theorem for the Laplace transform.
\end{abstract}

Given $r>0$, let $C=C([-r, 0], \mathbb{R})$ denote the Banach space of all continuous functions from $[-r, 0]$ into $\mathbb{R}$ equipped with the supremum norm,

$$
\|\phi\|=\sup _{-r \leq \theta \leq 0}|\phi(\theta)|, \quad \phi \in C .
$$

Consider the linear homogeneous difference equation

$$
D x_{t}=0,
$$

where the difference operator $D: C \rightarrow \mathbb{R}$ is given by

$$
D \phi=\phi(0)-\int_{-r}^{0} \phi(\theta) d \eta(\theta), \quad \phi \in C,
$$

and the symbol $x_{t} \in C$ is defined by $x_{t}(\theta)=x(t+\theta)$ for $\theta \in[-r, 0]$. Throughout the paper, we assume that the kernel $\eta:[-r, 0] \rightarrow \mathbb{R}$ is a nonconstant nondecreasing function such that $\eta(0)=0$ and $\eta$ is continuous from the left at each $\theta \in(-r, 0]$. The integral in (2) is a Riemann-Stieltjes integral.

With Eq. (1), we associate an initial condition of the form

$$
x_{0}=\psi, \quad \text { where } \psi \in C_{D}=\{\phi \in C \mid D \phi=0\} .
$$

The monotonicity and left continuity of $\eta$ at zero imply that $\operatorname{Var}_{[s, 0]} \eta=\eta(0)-$ $\eta(s) \rightarrow 0$ as $s \rightarrow 0^{-}$and hence the difference operator $D$ is atomic at zero (see [6] for a definition). By known existence theorems (see, e.g., [6. Chap. 12]), the initial value problem (1)-(3) has a unique solution on $[-r, \infty)$. We shall write $x(t)=x(t, \psi)$ for the unique solution of (1) and (3).

Received by the editors June 21, 2004.

2000 Mathematics Subject Classification. Primary 39A11; Secondary 34K40.

Key words and phrases. Difference equation, Cesàro summability, Lyapunov exponent, Laplace transform, Tauberian theorems.

This research was supported in part by the Hungarian National Foundation for Scientific Research (OTKA) Grant No. T 046929.

(C)2005 American Mathematical Society Reverts to public domain 28 years from publication 
The characteristic equation corresponding to (1) is

$$
\Delta(\lambda)=0, \quad \Delta(\lambda)=1-\int_{-r}^{0} e^{\lambda \theta} d \eta(\theta) .
$$

It is easy to show that under the above hypotheses on $\eta, \Delta(-\infty)=-\infty, \Delta(\infty)=1$ and

$$
\Delta^{\prime}(\lambda)=-\int_{-r}^{0} \theta e^{\lambda \theta} d \eta(\theta)>0 \quad \text { for all } \lambda \in \mathbb{R} .
$$

Consequently, the characteristic equation (4) has a unique (simple) real root $\lambda_{0}$.

Our aim in this note is to show that for every $\psi \in C_{D}$ the function $x(t, \psi) e^{-\lambda_{0} t}$ is Cesàro summable to a limit $l(\psi)$ which can be expressed explicitly in terms of the initial data $\psi$.

Theorem 1. Let the above hypotheses on $\eta$ hold. Then for every $\psi \in C_{D}$ the limit

$$
l(\psi)=\lim _{t \rightarrow \infty} \frac{1}{t} \int_{0}^{t} x(\tau, \psi) e^{-\lambda_{0} \tau} d \tau
$$

exists, and its value is given by

$$
l(\psi)=\frac{1}{\Delta^{\prime}\left(\lambda_{0}\right)} \int_{-r}^{0}\left(e^{\lambda_{0} \theta} \int_{\theta}^{0} \psi(\tau) e^{-\lambda_{0} \tau} d \tau\right) d \eta(\theta) .
$$

The proof of Theorem 1 will be based on the following Tauberian theorem for the Laplace transform (see [9, Chap. 8, Theorem 2.4] or [1, Satz 4, p. 512]).

Theorem 2. Suppose that $y:[0, \infty) \rightarrow \mathbb{R}$ is a continuous bounded function and let $\tilde{y}(s)$ denote its Laplace transform,

$$
\tilde{y}(s)=\int_{0}^{\infty} e^{-s \tau} y(\tau) d \tau \quad \text { for } s>0 .
$$

If

$$
\text { s } \tilde{y}(s) \rightarrow A \quad \text { for some } A \in \mathbb{R} \text { as } s \rightarrow 0^{+},
$$

then

$$
\frac{1}{t} \int_{0}^{t} y(\tau) d \tau \rightarrow A \quad \text { as } t \rightarrow \infty
$$

Proof of Theorem 1. Let $\psi \in C_{D}$ be arbitrary. Define

$$
y(t)=x(t, \psi) e^{-\lambda_{0} t} \quad \text { for } t \geq-r .
$$

From (1) and (2), we obtain for $t \geq 0$,

$$
y(t)=\int_{-r}^{0} y(t+\theta) e^{\lambda_{0} \theta} d \eta(\theta) .
$$

Choose $M>\max _{-r \leq t \leq 0}|y(t)|$. We shall show that

$$
|y(t)|<M \quad \text { for all } t \geq 0 .
$$

Otherwise, there exists $t_{1}>0$ such that

$$
|y(t)|<M \quad \text { for }-r \leq t<t_{1} \quad \text { and } \quad\left|y\left(t_{1}\right)\right|=M .
$$


From (10), it follows by well-known properties of the Riemann-Stieltjes integral

$$
\begin{aligned}
\left|y\left(t_{1}\right)\right| & \leq \int_{-r}^{0}\left|y\left(t_{1}+\theta\right)\right| e^{\lambda_{0} \theta} d \eta(\theta) \\
& =\int_{-r}^{-\delta}\left|y\left(t_{1}+\theta\right)\right| e^{\lambda_{0} \theta} d \eta(\theta)+\int_{-\delta}^{0}\left|y\left(t_{1}+\theta\right)\right| e^{\lambda_{0} \theta} d \eta(\theta)
\end{aligned}
$$

for $\delta \in(0, r)$. By virtue of (12), we have

$$
\int_{-r}^{-\delta}\left|y\left(t_{1}+\theta\right)\right| e^{\lambda_{0} \theta} d \eta(\theta) \leq M \int_{-r}^{-\delta} e^{\lambda_{0} \theta} d \eta(\theta)
$$

and

$$
\int_{-\delta}^{0}\left|y\left(t_{1}+\theta\right)\right| e^{\lambda_{0} \theta} d \eta(\theta) \leq M \int_{-\delta}^{0} e^{\lambda_{0} \theta} d \eta(\theta)
$$

We claim that if $\delta>0$ is sufficiently small, then the inequality in (14) is strict. Indeed, by the hypotheses on $\eta, \eta(-r)<\eta(0)$ and therefore $\eta(-r)<\eta(-\delta)$ for all sufficiently small $\delta$ by the left continuity of $\eta$ at zero. If $\delta$ is chosen in this way, then

$$
\begin{aligned}
& M \int_{-r}^{-\delta} e^{\lambda_{0} \theta} d \eta(\theta)-\int_{-r}^{-\delta}\left|y\left(t_{1}+\theta\right)\right| e^{\lambda_{0} \theta} d \eta(\theta) \\
& =\int_{-r}^{-\delta} f(\theta) d \eta(\theta) \geq \min _{-r \leq \theta \leq-\delta} f(\theta)[\eta(-\delta)-\eta(-r)]>0,
\end{aligned}
$$

where $f(\theta)=\left(M-\left|y\left(t_{1}+\theta\right)\right|\right) e^{\lambda_{0} \theta}>0$ for $-r \leq \theta \leq-\delta$ by the first (strict) inequality in (12). Thus, (14) holds with a strict inequality. Using this and (15) in (13), we obtain

$$
M=\left|y\left(t_{1}\right)\right|<M \int_{-r}^{-\delta} e^{\lambda_{0} \theta} d \eta(\theta)+M \int_{-\delta}^{0} e^{\lambda_{0} \theta} d \eta(\theta)=M \int_{-r}^{0} e^{\lambda_{0} \theta} d \eta(\theta)=M
$$

a contradiction. (We have used the fact that $\lambda_{0}$ is a root of (4).) Consequently, (11) holds and therefore the Laplace integral (7) converges for all $s>0$.

Taking the Laplace transform of (10) and changing the order of integration on the right-hand side, we find for $s>0$,

$$
\begin{aligned}
\tilde{y}(s) & =\int_{-r}^{0} e^{\left(\lambda_{0}+s\right) \theta}\left(\int_{0}^{-\theta} e^{-s(t+\theta)} y(t+\theta) d t+\int_{-\theta}^{\infty} e^{-s(t+\theta)} y(t+\theta) d t\right) d \eta(\theta) \\
& =\int_{-r}^{0} e^{\left(\lambda_{0}+s\right) \theta} \int_{\theta}^{0} e^{-s \tau} y(\tau) d \tau d \eta(\theta)+\int_{-r}^{0} e^{\left(\lambda_{0}+s\right) \theta} d \eta(\theta) \int_{0}^{\infty} e^{-s \tau} y(\tau) d \tau .
\end{aligned}
$$

Hence

$$
g(s) \tilde{y}(s)=h(s)
$$

where

$$
g(s)=1-\int_{-r}^{0} e^{\left(\lambda_{0}+s\right) \theta} d \eta(\theta)
$$

and

$$
h(s)=\int_{-r}^{0} e^{\left(\lambda_{0}+s\right) \theta} \int_{\theta}^{0} \psi(\tau) e^{-\left(\lambda_{0}+s\right) \tau} d \tau d \eta(\theta) .
$$


Since $\lambda_{0}$ is a root of (4), $g(0)=0$ and hence

$$
\frac{g(s)}{s} \longrightarrow g^{\prime}(0)=\Delta^{\prime}\left(\lambda_{0}\right) \quad \text { as } s \rightarrow 0
$$

by l'Hospital's rule. From this and (16), we obtain

$$
s \tilde{y}(s)=\frac{s}{g(s)} h(s) \longrightarrow \frac{h(0)}{\Delta^{\prime}\left(\lambda_{0}\right)}=l(\psi) \quad \text { as } s \rightarrow 0^{+}
$$

with $l(\psi)$ as in (6). By Theorem 2, this implies (5).

Remark. In the previous proof, the monotonicity of $\eta$ is used only to show the boundedness of the function $y(t)=x(t, \psi) e^{-\lambda_{0} t}, t \geq 0$. The same proof shows that if the kernel $\eta$ is merely of bounded variation on $[-r, 0]$ and we know a priori that a solution $x(t, \psi)$ of (1) with $\psi \in C_{D}$ is of order

$$
x(t, \psi)=O\left(e^{\lambda_{0} t}\right) \quad \text { as } t \rightarrow \infty,
$$

where $\lambda_{0}$ is a simple real characteristic value, then the Cesàro summability (5) holds with a limit $l(\psi)$ given by $(6)$.

For $\psi \in C_{D}$, the (Lyapunov) characteristic exponent $\alpha(\psi)$ of the solution $x(t, \psi)$ of (1) is defined by

$$
\alpha(\psi)=\inf \left\{\beta \in \mathbb{R} \mid x(t, \psi)=O\left(e^{\beta t}\right) \text { as } t \rightarrow \infty\right\} .
$$

The following simple corollary of Theorem 1 shows that for "almost all" solutions, $\alpha(\psi)=\lambda_{0}$.

Corollary 3. Under the hypotheses of Theorem $1, \alpha(\psi)=\lambda_{0}$ except possibly if $\psi$ belongs to a certain nowhere dense subset of the Banach space $C_{D}$ with the supremum norm.

Proof. $C_{D}$ is the kernel of the linear functional $D: C \rightarrow \mathbb{R}$. Since $D$ is continuous, its kernel is a closed subspace of $C$ and thus it is a Banach space.

The proof of the corollary will be complete if we prove the following two claims:

(i) If $\alpha(\psi) \neq \lambda_{0}$ for some $\psi \in C_{D}$, then $\psi$ belongs to the set

$$
\mathcal{N}=\left\{\phi \in C_{D} \mid l(\phi)=0\right\},
$$

the kernel of the linear functional $l: C_{D} \rightarrow \mathbb{R}$.

(ii) $\mathcal{N}$ is a nowhere dense subset of $C_{D}$.

Proof of (i). Let $\psi \in C_{D}$ such that $\alpha(\psi) \neq \lambda_{0}$. As shown in the proof of Theorem 1 , the function $x(t, \psi) e^{-\lambda_{0} t}$ is bounded for $t \rightarrow \infty$. Therefore $\alpha(\psi)$ cannot be greater than $\lambda_{0}$. Thus, $\alpha(\psi)<\lambda_{0}$ and hence $x(t, \psi) e^{-\lambda_{0} t}$ tends to zero (exponentially) as $t \rightarrow \infty$. Consequently, for every $\epsilon>0$ there exists $T>0$ such that $|x(t, \psi)| e^{-\lambda_{0} t}<\epsilon$ for $t \geq T$. This implies for $t \geq T$,

$$
\begin{aligned}
\left|\frac{1}{t} \int_{0}^{t} x(s, \psi) e^{-\lambda_{0} s} d s\right| & \leq \frac{1}{t} \int_{0}^{T}|x(s, \psi)| e^{-\lambda_{0} s} d s+\frac{1}{t} \int_{T}^{t}|x(s, \psi)| e^{-\lambda_{0} s} d s \\
& \leq \frac{1}{t} \int_{0}^{T}|x(s, \psi)| e^{-\lambda_{0} s} d s+\frac{\epsilon(t-T)}{t} .
\end{aligned}
$$

Letting $t \rightarrow \infty$ in the last inequality and using (5), we get $|l(\psi)| \leq \epsilon$. Since $\epsilon>0$ was arbitrary, $l(\psi)=0$ and $\psi \in \mathcal{N}$.

Proof of (ii). Since $l: C_{D} \rightarrow \mathbb{R}$ is continuous (see (6)), $\mathcal{N}$ is a closed subset of $C_{D}$. It remains to show that $\mathcal{N}$ has empty interior in $C_{D}$. Let $\psi \in \mathcal{N}$. For $\epsilon>0$, 
let $\psi_{\epsilon}=\psi+\epsilon e_{\lambda_{0}}$, where $e_{\lambda_{0}} \in C$ is defined by $e_{\lambda_{0}}(\theta)=e^{\lambda_{0} \theta}$ for $-r \leq \theta \leq 0$. Since $\lambda_{0}$ is a root of the characteristic equation (4), $e_{\lambda_{0}}$ and hence each $\psi_{\epsilon}$ belongs to $C_{D}$. Clearly, $\psi_{\epsilon} \rightarrow \psi$ in $C_{D}$ as $\epsilon \rightarrow 0^{+}$, while $l\left(\psi_{\epsilon}\right)=l(\psi)+l\left(\epsilon e_{\lambda_{0}}\right)=\epsilon l\left(e_{\lambda_{0}}\right)=\epsilon \neq 0$. This implies that $\psi$ does not belong to the interior of $\mathcal{N}$. Since $\psi \in \mathcal{N}$ was arbitrary, $\mathcal{N}$ has empty interior in $C_{D}$.

Remark. The property that for most solutions the asymptotic behavior can be determined in terms of a simple real dominant characteristic value has been observed in many other linear systems (see, e.g., [2], 3], 4, [5], 7], [8] for related results).

Eq. (1) includes as a special case the equation

$$
x(t)=\sum_{i=0}^{k} a_{i} x\left(t-\tau_{i}\right),
$$

where the coefficients $a_{i}$ are nonzero real numbers and $0<\tau_{0}<\tau_{1}<\cdots<\tau_{k}$. In this case, $r=\tau_{k}$ and the kernel $\eta$ in the representation of $D$ is given by

$$
\eta(\theta)=\sum_{i=0}^{k} a_{i} H\left(\theta+\tau_{i}\right), \quad-r \leq \theta \leq 0,
$$

where $H(s)=0$ for $s>0$ and $H(s)=-1$ for $s \leq 0$. Our hypotheses on $\eta$ hold if $a_{i}>0$ for each $i=0,1, \ldots, k$. The list of the above symbols is the following:

$$
\begin{aligned}
D \phi & =\phi(0)-\sum_{i=0}^{k} a_{i} \phi\left(-\tau_{i}\right), \\
C_{D} & =\left\{\phi \in C \mid \phi(0)=\sum_{i=0}^{k} a_{i} \phi\left(-\tau_{i}\right)\right\}, \\
\Delta(\lambda) & =1-\sum_{i=0}^{k} a_{i} e^{-\lambda \tau_{i}}, \\
l(\psi) & =\frac{\sum_{i=0}^{k} a_{i} e^{-\lambda_{0} \tau_{i}} \int_{-\tau_{i}}^{0} \psi(t) e^{-\lambda_{0} t} d t}{\sum_{i=0}^{k} a_{i} \tau_{i} e^{-\lambda_{0} \tau_{i}}} .
\end{aligned}
$$

Finally, let us mention a recent result due to Philos and Purnaras [8] which is relevant to our study. They have considered Eq. (17) under the additional assumption that the delays $\tau_{i}$ are commensurable in the sense that for each $i=1,2, \ldots, k$,

$$
\tau_{i}=m_{i} \tau_{0} \quad \text { for some positive integer } m_{i}
$$

and the corresponding characteristic equation

$$
1=a_{0} e^{-\lambda \tau_{0}}+\sum_{i=1}^{k} a_{i} e^{-\lambda m_{i} \tau_{0}}
$$

has a real root $\lambda_{0}$ with the property

$$
\sum_{i=1}^{k}\left|a_{i}\right|\left(m_{i}-1\right) e^{-\lambda_{0} m_{i} \tau_{0}}<1 .
$$


In [8, Theorem 1.2], they show that under these hypotheses for every $\psi \in C_{D}$ with $C_{D}$ as in (19) the solution $x(t, \psi)$ of (17) satisfies the asymptotic relation

$$
\int_{t-\tau_{0}}^{t} x(s, \psi) e^{-\lambda_{0} s} d s \rightarrow L(\psi) \quad \text { as } t \rightarrow \infty,
$$

where

$$
L(\psi)=\frac{\int_{-\tau_{0}}^{0} e^{-\lambda_{0} s} \psi(s) d s+\sum_{i=1}^{k} a_{i} e^{-\lambda_{0} m_{i} \tau_{0}} \int_{-\tau_{i}}^{-\tau_{0}} e^{-\lambda_{0} s} \psi(s) d s}{1+\sum_{i=1}^{k} a_{i}\left(m_{i}-1\right) e^{-\lambda_{0} m_{i} \tau_{0}}} .
$$

Note that in $[8$ the characteristic equation is written in a form which is obtained from (23) by the transformation $\mu=e^{\lambda \tau_{0}}$.

The limit relation (25) is stronger than (5). This is a consequence of the following elementary result applied to the function $f(t)=\int_{0}^{t} x(s, \psi) e^{-\lambda_{0} s} d s$.

Lemma 4. If $\tau_{0}>0$ and $f: \mathbb{R}_{+} \rightarrow \mathbb{R}$ is bounded on each bounded subset of $\mathbb{R}_{+}$, then the existence of the (finite) limit $L=\lim _{t \rightarrow \infty}\left(f\left(t+\tau_{0}\right)-f(t)\right)$ implies the existence of the limit $l=\lim _{t \rightarrow \infty} f(t) / t$. Moreover, $l=L / \tau_{0}$.

Since we could not find a reference for Lemma 4, we present its proof.

Proof of Lemma 4. Let $S=\lim \sup _{t \rightarrow \infty} f(t) / t$. Then there exist $t_{n} \rightarrow \infty$ such that $f\left(t_{n}\right) / t_{n} \rightarrow S$ as $n \rightarrow \infty$. The existence of the limit $L=\lim _{t \rightarrow \infty}\left(f\left(t+\tau_{0}\right)-f(t)\right)$ implies that for every $\epsilon>0$ there exists $T>0$ such that

$$
L-\epsilon<f\left(t+\tau_{0}\right)-f(t)<L+\epsilon \quad \text { for } t \geq T .
$$

For all sufficiently large $n$, we have $t_{n}>T+\tau_{0}$ and hence $t_{n}=T_{n}+k_{n} \tau_{0}$ for some $T_{n} \in\left[T, T+\tau_{0}\right)$ and positive integer $k_{n}$ ( $k_{n}$ is the greatest integer part of $\left.\left(t_{n}-T\right) / \tau_{0}\right)$. This, together with $(27)$, implies for all large $n$,

$$
\frac{f\left(t_{n}\right)-f\left(T_{n}\right)}{t_{n}-T_{n}}=\frac{1}{k_{n} \tau_{0}} \sum_{i=0}^{k_{n}-1}\left(f\left(T_{n}+(i+1) \tau_{0}\right)-f\left(T_{n}+i \tau_{0}\right)\right)<\frac{L+\epsilon}{\tau_{0}} .
$$

Taking into account that both sequences $\left\{T_{n}\right\}$ and $\left\{f\left(T_{n}\right)\right\}$ are bounded (for $T_{n} \in$ $\left[T, T+\tau_{0}\right)$ and $f$ is bounded on $\left[T, T+\tau_{0}\right)$ by the hypothesis), it follows that

$$
\frac{f\left(t_{n}\right)-f\left(T_{n}\right)}{t_{n}-T_{n}}=\left(\frac{f\left(t_{n}\right)}{t_{n}}-\frac{f\left(T_{n}\right)}{t_{n}}\right)\left(1-\frac{T_{n}}{t_{n}}\right)^{-1} \longrightarrow S \quad \text { as } n \rightarrow \infty .
$$

Consequently, letting $n \rightarrow \infty$ in (28), we get $S \leq(L+\epsilon) / \tau_{0}$. It can be shown in a similar manner (using the first inequality in (27)) that if $I=\liminf _{t \rightarrow \infty} f(t) / t$, then $I \geq(L-\epsilon) / \tau_{0}$. Since $\epsilon>0$ was arbitrary, we conclude that $L / \tau_{0} \leq I \leq S \leq L / \tau_{0}$ and hence $I=S=L / \tau_{0}$. This implies the desired conclusion.

According to Lemma 4, the existence of the limit (25) implies that (5) holds with $l(\psi)=L(\psi) / \tau_{0}$. (The latter equality can also be checked directly using formulae (21), (22), (23) and (26).) Consequently, for the above specific class of (17) with properties (22) and (24), conclusion (25) of Philos and Purnaras [8] is stronger than the Cesàro summability (5). On the other hand, in the case of positive coefficients, in contrast with [8, Theorem 1.2], we require neither the "smallness" condition (24) nor the rather restrictive condition (22) that the delays in (17) are commensurable. 


\section{REFERENCES}

[1] G. Doetsch, Handbuch der Laplace-Transformation I, Verlag Birkhäuser, Basel, 1950 (in German). MR0043253(13:230f)

[2] R. D. Driver, Some harmless delays, Delay and Functional Differential Equations and Their Applications (Proc. Conf., Park City, Utah, 1972), Academic Press, New York, 1972, pp. 103119. MR0385277 (52:6141)

[3] R. D. Driver, D. W. Sasser, and M. L. Slater, The equation $x^{\prime}(t)=a x(t)+b x(t-\tau)$ with "small" delay, Amer. Math. Monthly 80 (1973), 990-995. MR0326104 (48:4449)

[4] R. D. Driver, G. Ladas, and P. N. Vlahos, Asymptotic behavior of a linear delay difference equation, Proc. Amer. Math. Soc. 115 (1992), 105-112. MR1111217 (92i:39004)

[5] M. V. S. Frasson and S. M. Verduyn Lunel, Large time behaviour of linear functional differential equations, Integral Equations Operator Theory 47 (2003), 91-121. MR2015849 (2004j:34141)

[6] J. Hale, Theory of Functional Differential Equations, Springer-Verlag, New York, 1977. MR 0508721 (58:22904)

[7] I.-G. E. Kordonis and Ch. G. Philos, On the behavior of the solutions for linear autonomous neutral delay difference equations, J. Differ. Equations Appl. 5 (1999), 219-233. MR1697057 (2000b:39001)

[8] Ch. G. Philos and I. K. Purnaras, An asymptotic result for some delay difference equations with continuous variable, Advances in Difference Equations 2004:1 (2004), 1-10. MR2059199

[9] D. V. Widder, Introduction to Transform Theory, Academic Press, New York, 1971.

Department of Mathematics and Computing, University of Veszprém, P. O. Box 158, 8201 Veszrpém, Hungary

E-mail address: pitukm@almos.vein.hu 